\title{
Gravitational Lens Images Generated by Cosmic Strings
}

\author{
Mikhail V. Sazhin ${ }^{1, *}$, Olga S. Sazhina ${ }^{1}$, Massimo Capaccioli ${ }^{2,3,5,6}$, Giuseppe Longo ${ }^{2,3,5}$, Maurizio \\ Paolillo $^{3,4}$, and Giuseppe Riccio, \\ ${ }^{1}$ Sternberg Astronomical Institute, Moscow State University, University pr. 13, Moscow, Russia \\ ${ }^{2}$ INAF - Osservatorio Astronomico di Capodimonte, via Moiariello 16, 80131, Napoli Italy \\ ${ }^{3}$ Department of Physical Sciences, University of Napoli Federico II, via Cinthia 9, 80126 Napoli, Italy \\ ${ }^{4}$ INFN - Napoli Unit, Dept. of Physical Sciences, via Cinthia 9, 80126, Napoli, Italy \\ ${ }^{5}$ Department of Astronomy, California Institute of Technology, Pasadena, 91125 CA, USA \\ ${ }^{6}$ MECENAS, Università di Napoli Federico II and Università di Bari, Italy
}

\begin{abstract}
In this review paper the current understanding on the properties of cosmic strings is shortly outlined with special emphasis on the observational signatures which can be expected both in the optical, through gravitational lensing, and in the radio, through anisotropies in the cosmic microwave background. The experience gathered during the long term investigation of the former candidate CSL-1 is also shortly summarized.
\end{abstract}

Keywords: Cosmology, cosmic string, quasars, gravitational lensing, the CMBR anisotropy.

\section{COSMIC STRINGS: GENERALITIES AND THEIR ROLE IN PARTICLE PHYSICS}

The topological defects of space-time known as cosmic strings were introduced in the late seventies by Kibble [1] and have been extensively discussed over the past decades by several authors $[2,3]$.

Their existence finds support in a large class of early Universe models based on the superstring theory [4-6]. Cosmic strings are in fact predicted by compactification models, in theories with extended additional dimensions, and are easily justified within the current inflationary scenarios. It is important to stress that in comparision with other topological defects such as monopoles, domain walls, textures, etc., cosmic strings are the most likely to exist.

Nowadays, inflation finds a widespread consensus within the cosmological community and is in good agreement with observational astronomical data. On the other end, the braneantibrane inflationary mechanism is the most natural model arising from superstring theories (being one of the most promising to achieve a full description of the Universe at Planckian time-scales) theory tells us that, within this mechanism, inflation ends when the brane and the antibrane annihilate. This process is likely to generate an extremely rich spectrum of post-inflationary remnants. Cosmic strings are among the most likely remnants with a rather rich spectrum.

Without any doubt, the discovery of any of such objects would lead to a quantum leap in many fields of fundamental physics and, more specifically, in understanding the rules of GUT and the processes occurring in the pre-inflationary Universe. The linear density of a string (or its tension)

*Address correspondence to this author at the Sternberg Astronomical Institute, Moscow State University, University pr. 13, Moscow, Russia; Tel: +7 49593950 06; Fax: +7 49593288 41;

E-mail: miksazhin@yandex.ru depends in fact on the symmetry breaking scale. The specific equation which links these two values depends both on the model of phase transition and on the specific form of the scalar field potential. In the case of a global $U(1)$ string (i.e. the simplest case with winding number $n=1$ ), the equation is, [7]:

$\mu \approx \varepsilon_{\text {vac }} \delta_{H}^{2}\left[\mathrm{~g} \cdot \mathrm{cm}^{-1}\right]$

where $\varepsilon_{v a c}$ is the energy density of the false vacuum and $\delta_{H}$ is the Compton wavelenght of the Higgs boson. Here $\mu$ has dimension of linear density.

The most relevant and, what is more important, model independent feature of cosmic strings is that they are one dimensional objects with diameter of the order of Planckian size of $10^{-33} \mathrm{~cm}$. The microscopic characteristic of the string is the diameter, which is of the order of the square root of $\mu^{-1}$. Using Planck units, $\mu=1$ corresponds in usual units to $1.4 \cdot 10^{28} \mathrm{~g} \mathrm{~cm}^{-1}$ and the diameter of the string would be of the order of $10^{-33} \mathrm{~cm}$. An estimated lenght which is compatible with the observable part of the Universe. During the evolution strings can intersect, passing through one another or exchanging their partners (intercommuting), [7]. The fraction of straight strings and loops is model dependent. For instance, simulations done in the Goto-Nambu model show that the fraction of closed loops is around $20 \%$, but it may increase up to $50 \%$ in other models [6]. In any case, if strings do exist, theory predicts that the number of long strings is large enough to justify a systematic search. It needs to be stressed that the velocity of long strings is smaller than that expected for shorter segments [8], but the expected value of string velocity is large enough to produce a distinguishable discontinuity in the CMBR.

The space-time in the presence of cosmic string is conical, and a 2D slice of the conical space is presented on Fig. (1). It is flat except than in the vertex of the cone which coincides with the position of one point of the cosmic string 


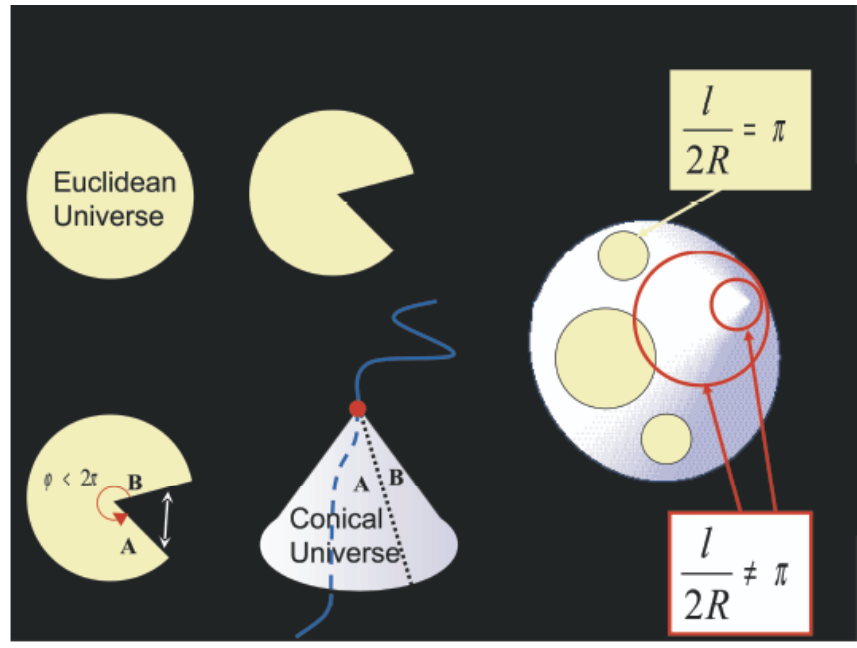

Fig. (1). The transformation of the 2D Euclidean space into 2D conical space. In the left upper corner, the circle of the Euclidean space is drawn. By cutting out a sector, only a part of the space is left and the conical space is obtained by joining the cuts. The vertex of the cone is the location of the cosmic string. The space is Euclidean overall except that in the vertex.

in 3D space. Fig. (1) depicts how the 2D Euclidean space transforms into a 2D conical one.

As we already mentioned, theory allows for the existence of closed cosmic strings (loops), but in what follows we shall consider only long straight strings. It is important to notice that these cosmic strings have no ends and that formally speaking, their ends are the two intersection points with the particle horizon. The reason why we shall consider only straight string is simple, for astronomical constrains, these objects are much easier to detect than loops.

Recent string network simululations have posed upper limits to the number of cosmic strings which could exist in our Universe. The analysis of the WMAP CMBR data by [9-12] provides us with an upper limit to the linear density of a cosmic string.

As we shall see, the signature left by cosmic strings on the CMBR anisotropy depends both on the linear density of the cosmic string and on the number of cosmic string falling inside the particle horizon of our Universe. The number of cosmic strings depends on the assumed string formation model, hence it is strongly model dependent. We shall

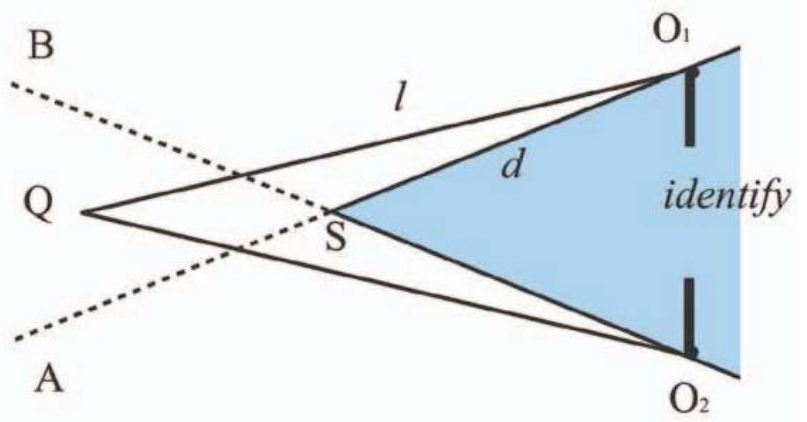

Fig. (2). The formation of two images of background point-like source, which is lensed by a cosmic string. concentrate on the model of very rarified network. A choice which is supported by the fact that observations prove that in our Universe only few cosmic strings exist, if any. The proof being the negative detection result received by independent collaborations till present time.

\section{OBSERVATIONAL SIGNATURES OF COSMIC STRINGS}

From the observational point of view, the most effective way to discover the existence of a non-closed cosmic string is the detection of what we define as a sort of "milky way" of gravitationally lensed background sources which look like a chain of gravitational lensed pairs. Since a cosmic string has a long structure, it is more probable to observe a set of such pairs, rather than isolated pairs. It seems that for significantly curved string, instead of pairs more images will appear, but we do not take into account these possibility seems they are more rare.

In order to better understand the gravitational lens effects of a string, let us refer to Fig. (1), 1 and remind that in presence of a cosmic string, the 4D space-time becomes conical. The photons from a background source move around the string and, by circum-navigating it, they form two images on both sides of the string itself. Since along the two trajectories the space is flat, there is no gravitational attraction exerted by the string on the photons and no distortion is introduced.

However, in spite of the fact that the metric is locally flat, the global properties of the space-time are not Minkowskian but conical, Fig. (1), and a complete turn around the position of the string gives a total angle $\varphi$ smaller than $2 \pi$, while the difference $2 \pi-\varphi$ is the so-called "deficit angle" $\Delta \theta$ which defines the lensing properties of the string.

Its value depends on the string energy (or string strenght, or string linear density).

$\Delta \theta=8 \pi G \mu$,

where $\mu$ is the linear density of a cosmic string. In theories which do not contradict current astrophysical observations, for strings forming after the inflation, the deficit angle is $\sim 2$, which corresponds to an energy scale of false vacuum around $10^{16} \mathrm{GeV}$.

Now, since a cosmic string is an extended object, the light from all objects laying in the background within an angular distance from the string smaller than the deficit angle will be lensed. The final effect being that along the path of the string will appear a myriad of double images, the so called "milky way".

A second aspect of gravitational lensing by a cosmic string is that, at a difference with what happens for compact lenses, the images will be virtually undistorted due to the conical nature of the space time. The only distortion which can arise are sharp edges in the fainter isophotes of an extended background source. We shall return to this aspect in section 4, where we shall discuss the case of CSL-1. Here we wish to stress that, in contrast to what happens in other lensing models, the lensing by a cosmic string does not introduce any amplification of an individual image from the 
background source. In the case of a point-like source, such as QSOs, falling inside the cosmic string strip, the observer will see two identical images of the source with defined position [13]. As long as the source is point-like and the photon beams move in a quasi-Euclidean space, the two images will have identical optical properties. Hence, apart from a small frequency shift due to the motion of the string, there will be two identical images, which are neither distorted, not amplified. Deviation from this idealized pattern can be caused by string curvature, by masses distributed near the line of sight, or by unequal time delays for the two images. This time delay is aproximately a few years for GUT scale strings. In fact, it is possible that there is very large magnification of the image due to caustics, which could be formed by small string loops of the order of a separation between images [7]. In this case, which unfortunately is very model dependend, the image configuration and luminosity can have an observable time variability.

The method of searching for gravitational lensing events is based on optical observations. Modern optical surveys cover only $1 / 6$ part of the whole sky and their redshift depth is not more than $z=2-3$, although some optical objects have redshift up to $\mathrm{z}=7$. Therefore, approximately only $3 \%$ of the existing strings can be detected with this method (if we suppose that they are distributed in an homogeneous way). Taking into account the predicted number of cosmic strings (we discuss it below) it was estimated that gravitational lensing events are very rare ones. This estimate can explain the unsuccessful previous attempts and shows that additional methods for cosmic string search are urgently required because even utilization of ultra deep galaxy surveys is not enough.

A very important cosmic string signature is the effects they induce on the CMBR, where they can create linear discontinuities which translate into anisotropic signals overimposed on the CMBR [14-16]. In order to produce such signatures, the cosmic strings must move with velocities comparable to the speed of light. The discontinuity produced by a cosmic string in the CMBR can be written as:

$$
\frac{\delta T}{T}=8 \pi G \mu \gamma \frac{v}{c}
$$

where $\gamma$ is lorentz factor, $v$ is the transverse velocity with respect to the line of sight. One can also present this equation in a more appropriate form:

$\frac{\delta T}{T}=27 \mu K \frac{\Delta \theta}{2^{\prime \prime}} \frac{v}{0.9 c}$

This equation is very convenient to estimate the value of discontinuites for different values of the deficit angle.

The search for signatures in the CMBR appears to be a very promising due to two main reasons. First, this method operates with one of the model independent properties of cosmic strings, namely their possibility to form conical space-time. Second, the red shift depth of radio surveys is $\mathrm{z}=1000$ and covers the whole sky. Therefore using the WMAP 5-years data on the CMBR anisotropy, we can search for cosmic strings in the whole volume inside the surface of last scattering and can detect $100 \%$ of the strings and not the $3 \%$ estimated above for optical surveys.
Alternatively, a negative detection can disprove the existence of cosmic strings over a wide range of allowed parameters.

As far as we start to consider the moving string, several words on the gravitational lens features of these strings are necessary. In this case the Einstein strip will be nonstationary, and the position of strip on the sky will be changed.

The details of special string motion (i.e. the system of string segments moving not perpendicularly to the line of sight) is discussed in [7]. We are not considering these cases, but rather the simplest model of long straight string, moving in one plane and perpendicular to the line from the observer to the position of a string.

\section{THE NUMBER OF COSMIC STRINGS AND THE PROBABILITY OF THEIR OBSERVATION}

The background space-time we consider here corresponds to standard cosmological $\Lambda \mathrm{CDM}$ model with the FRW metric. The scale factor $a(t)$ of the metric is determined by the Friedmann equation. We also choose the WMAP recommended global cosmological parameters: $H_{0}=72 \mathrm{~km} / \mathrm{s} / \mathrm{Mpc}$ is the present value of the Hubble parameter, $a\left(t_{0}\right)=a_{0}=1$ is the present value of the scale factor, $\Omega_{\Lambda}$ refers to the cosmological constant. The values we shall use here are $\Omega_{m}=0.27, \Omega_{\Lambda}=0.73$.

Taking into account the usual determination of the distance to an object in the expanding Universe as

$$
l=c \int_{t_{e}}^{t_{o}} \frac{d t}{a(t)}
$$

where $t_{e}$ is epoch when the photons were emitted, $t_{o}$ is the present epoch, and by substituting $t_{e}=0$ one obtains the distance to particle horizon as:

$l_{p h}=3.4 \frac{c}{H_{0}}$

in the standard $\Lambda \mathrm{CDM}$ model.

One can also redefine $l$ in terms of red shift instead of time. In this redifinition the equation for the distance becomes

$$
l(z)=\frac{c}{H_{0}} \int_{0}^{z} \frac{d z}{\sqrt{\Omega_{m}(1+z)^{3}+\Omega_{\Lambda}}}
$$

This form for the cosmological distance is more convenient to perform a probabilistic analysis.

The distance betwen an observer and a cosmic object is a function of the redshift of the object and of the cosmological parameters. In order to estimate the probability to observe a string we suppose that the most distant object we are able to observe is at $z=7$. In this case we can calculate the radius of the sphere in which all avaible optical objects are:

$l_{7}=0.64 \cdot l_{p h}$

Let us now assume that there is a straight string inside the particle horizon sphere, then the probability that the string is 
inside the optical radius is proportional to the optical sphere volume:

$p=l_{7}^{3}=0.25$

where $l_{7}$ is the radius of this sphere in units of the distance to the particle horizon. Therefore only one string out of four is inside the sphere of optical sources. A detailed calculation of the probability to find a string and of the number of expected lenses can be found in [17-19]. In this case the string just "touches" the sphere of optical sources and the angular size of a string turns out to be $\sim 100^{\circ}$. If the redshift of optical source sphere is closer, then the size of straight string has to be much larger.

In the case $z=2$ the sphere radius is:

$l_{2}=0.38 \cdot l_{p h}$,

and the probability is $p=0.05$. In this case only one string out of twenty is inside the sphere and the angular size of a string is around $\sim 134^{\circ}$. The angular size of straight string is very important since it defines the scale of the signatures left in the radiomaps.

To observe gravitational lensing effect on a cosmic string or a cosmic superstring the number of strings must be larger than four in the observable Universe, because, as we mentioned above, only one string out of four is inside the sphere of optical sources. In order to derive the detection probability we must derive first the number of string inside the particle horizon which is model dependent. If we restrict ourselves to long strings alone we can use the value derived by [20] $\left(10^{3} \div 10^{4}\right)$.

By using such figures we derive that at least 50 strings should be closer than $z<2$ and astronomers should be able to detect at least a dozen of "new milky ways". Even though the task of finding them is rather complex due to limitations introduced by the faintness of the sources and by confusion problems, a hint on their positions can be obtained from the anisotropy of the CMBR.

\section{CSL-1 AND THE HISTORY OF ITS INVESTIGATION}

In 2003 a peculiar object named Capodimonte-Sternberg lens N.1 or CSL-1 located at $R A=12^{h} 23^{m} 30^{s}$, $D E C=-12^{\circ} 38^{\prime} 57^{\prime \prime}$, was discovered in the OACDF [21] by an international collaboration, [22]. CSL-1, is a double extended source laying in a low density field and with the two components separated by $\sim 1.9$ arcsec. Both components are well resolved (they are extended) and have roundish and identical shapes. Low resolution spectroscopy showed that both components are at a redshift of $0.46 \pm 0.008$, while photometry (both global properties and luminosity profiles) matched those of giant elliptical galaxies at medium redshift. Visual inspection, comparison of main absorption lines, the ratio of the spectra of the two components and their cross-correlation, showed that the spectra of the two components, altough in the limited spectral range covered by the original spectra, were identical at a $\sim 99 \%$ confidence level. The separation, compatible with the predictable deficit angle for a cosmic string, and the overall appearance of both optical images and spectra, arose the suspect that it could be the first case of gravitational lensing by a cosmic string. The other possible explanation, that of a rare chance alignment of two identical giant ellipticals at the same redshift seemed very unlikely due to a series of odd circumstances: the two ellipticals should have been relatively near, $=11 \mathrm{kpc}$, each other, and in spite of this, undistorted, with identical (within the errors) spectra, and residing in a relatively low density environment (no other galaxies could be detected in the same region). Even though at the end it was proved that, in spite of all odds, this was the case [23], the detailed study of this anomalous object allowed to investigate in some detail another aspect of the expected observational effects induced by cosmic strings $[13,17,22,24]$.

In fact, as it has already been mentioned above, one of the main characteristics of lensing by a cosmic string is that it does not introduce distortions. In order to try to disentangle between these two possible explanations, extensive simulations were needed, and they proved that the only way to clarify the nature of the object was to obtain high angular resolution images of the object. These simulations showed that lensing of an extended background object by a cosmic strings would introduce sharp edges in the low light level isophotes. In average seeing conditions, these sharp edges are rounded off and therefore high resolution data are needed. Fig. (3) shows the simulated sharp edges of a background source. Depending on the impact parameter of source with respect to the string, the cuts are different, they tend to zero in symmetric case.

In Fig. (4) we see the actual image of CSL-1 obtained with the ACS camera on HST. One can compare it with the simulated images of how CSL-1 should have appeared in the case it were a case of lensing by cosmic string. As it can be clearly seen, no sharp edges are presented and, what is even more relevant the shapes of the fainter isophotes are different and seem to indicate that some degree of interaction is presented. The most likely interpretation of CSL-1 is therefore that of two elliptical galaxies in an early state of interaction which may eventually lead to a dry merger.

Fig. (3) gives an example of what would happen to a circular source lensed by a string, notice that the sharp cut (edge) introduced by the string is clearly visible. The image of extended source produced by a cosmic string is characterized by special features which are unique to cosmic strings (for a detailed discussion see [23]). First of all we wish to stress that any extragalactic object can be considered as extended in comparison with cosmic string. In fact, while the strip of cosmic string lensing is defined by its linear density $\mu$, the width of the string itself is determined by the Compton wavelengths of the Higgs and gauge bosons $\left(10^{-9} \div 10^{-19} \AA\right)$. The gravitational lensing properties of a cosmic string can be easily understood as it follows: each pixel of the extended source which falls inside the string strip is displayed on the other side of the string, if it falls outside, it will be cut away producing sharp isophoteal edges on the image of the source.

Figs. $(\mathbf{5}, \mathbf{6}, \mathbf{7})$ show examples of what would happen to different sources lensed by a string. 


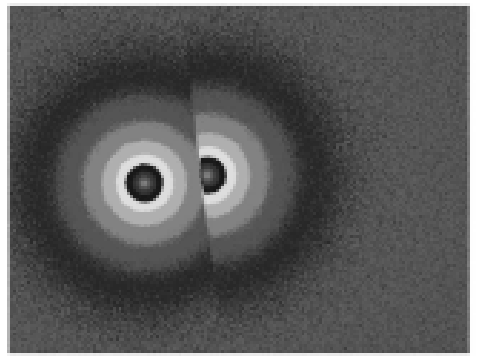

(a)

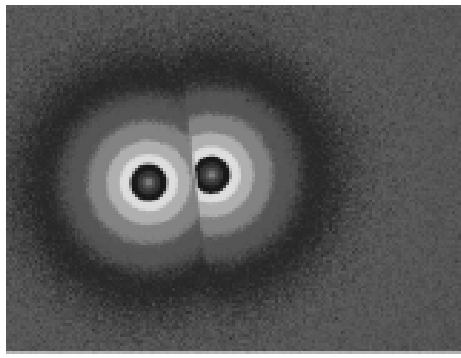

(c)

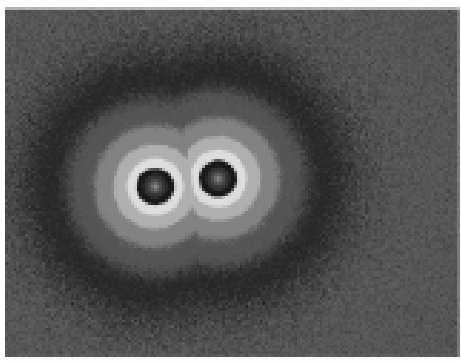

(e)

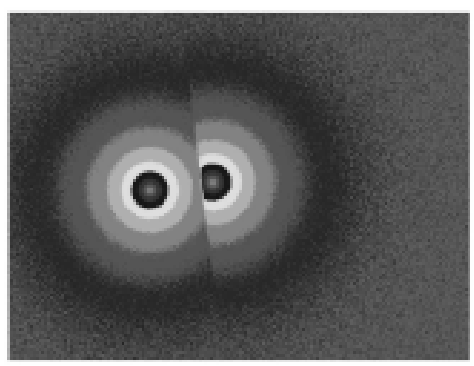

(b)

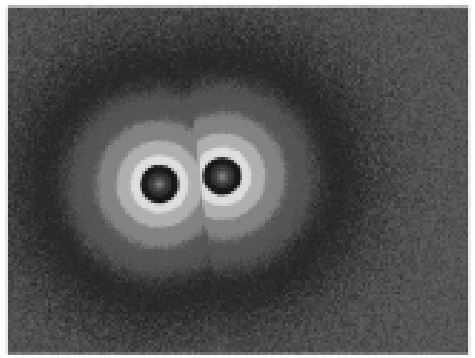

(d)

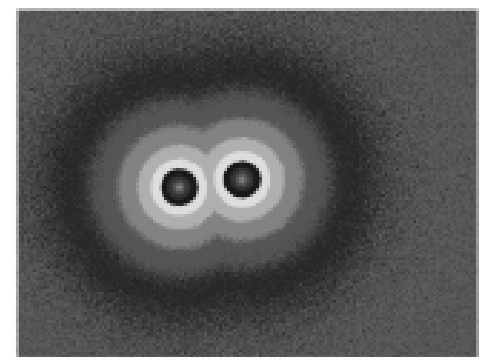

(f)

Fig. (3). The examples of sharp edges in different position of a background sources relative to the string strips. The CSL-1 case corresponds to the 'e' panel which is almost symmetric.

\section{CONCLUSIONS}

Cosmic strings are expected to produce well defined and unambiguous observational features. In optical surveys these features are of two types: chains of lensed galaxies and quasars and sharp edges in well resolved galactic images. In the CMBR, cosmic strings would introduce well defined steplike discontinuities. We therefore suggest that the most effective approach to the search of cosmic string would be first to search for CMBR signatures and then perform optical follow-up's. In the CMBR the anisotropy induced by a cosmic string would appear as a sequence of zones of decreased and increased temperature: first a cold spot in front of the moving string, then a step-like jump followed by an hot spot and, finally, a second cold spot [16]. The structure of such temperature fluctuation is virtually independent on the cosmic string parameters, since only the spot width and the temperatures at the local maximum and local minima are affected by the position of the string with respect to the observer, by the string velocity and direction, and by the string linear density. But the structure always remains the same.

For a cosmic string with deficit angle comprised between 1 and 2 arcsec, and transverse velocity close to the speed of light, the amplitude of the expected anisotropy varies from 15 to $30 \mu \mathrm{K}$.

If we shall find string candidates analizing the CMBR anisotropy data, then among all detected candidates we have to choose the strings with angular size larger than 100 in order to be sure that they are inside the sphere of optical sources $z<7$. Such candidates are expected to produce also the gravitational lensing effects which have been described in the previous paragraphs. 


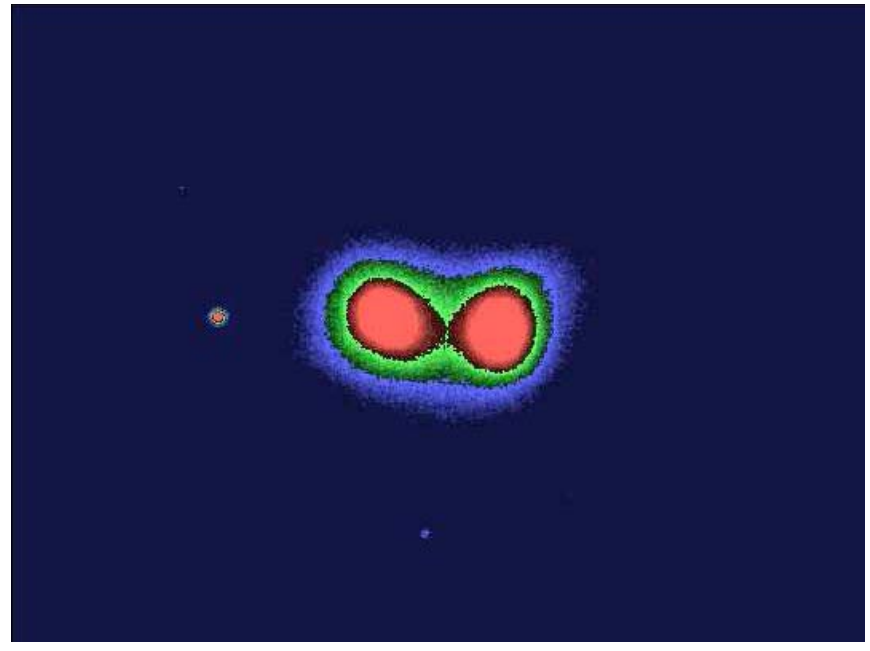

Fig. (4). The actual HST image of CSL-1.

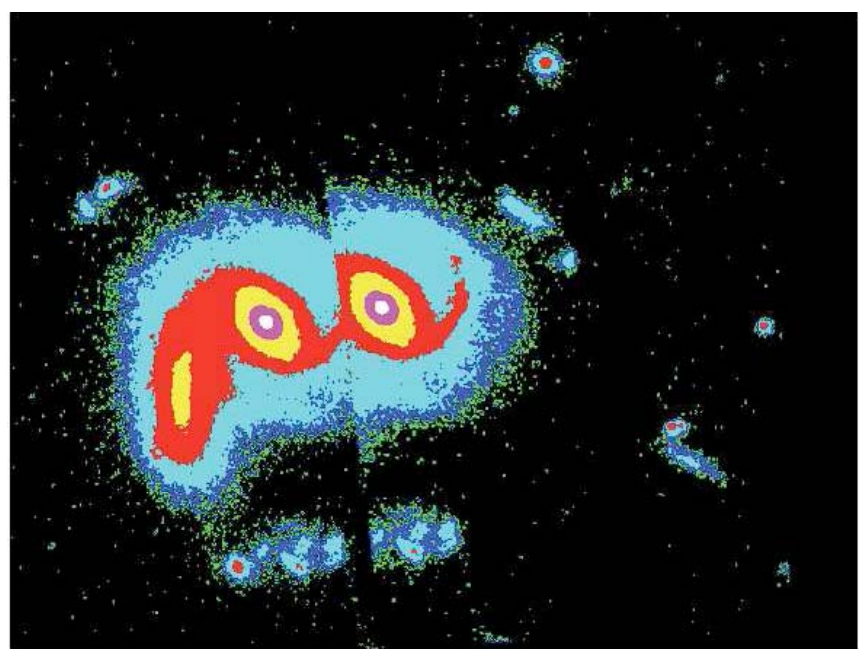

Fig. (5). First example of a galaxy lensed by acosmic string.

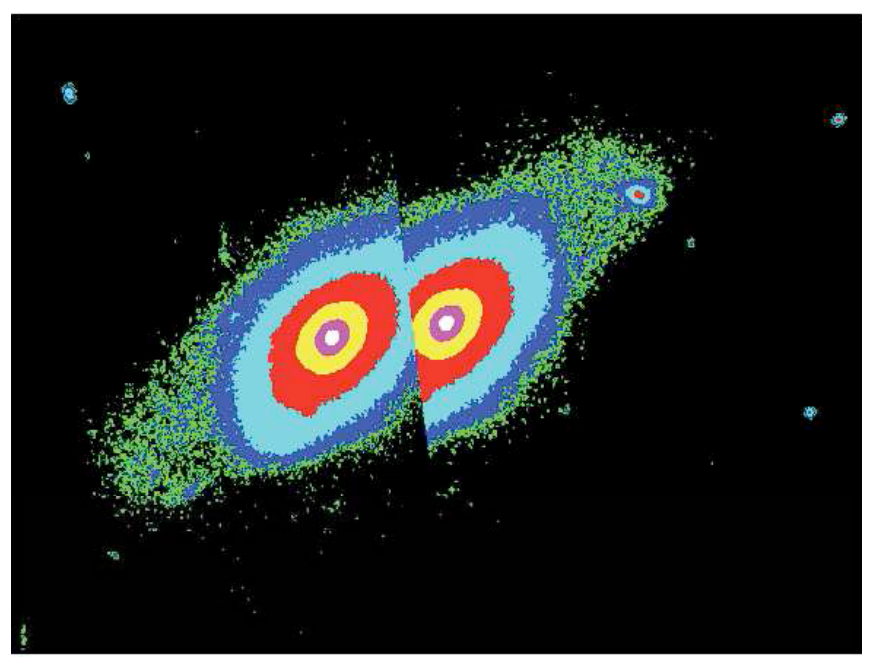

Fig. (6). Second example of a galaxy lensed by acosmic string.

Therefore, at the moment, theory has set the observational scenario and constrained the observational tests which need to be pursued in order to prove or disprove the existence of cosmic strings. The detection of such

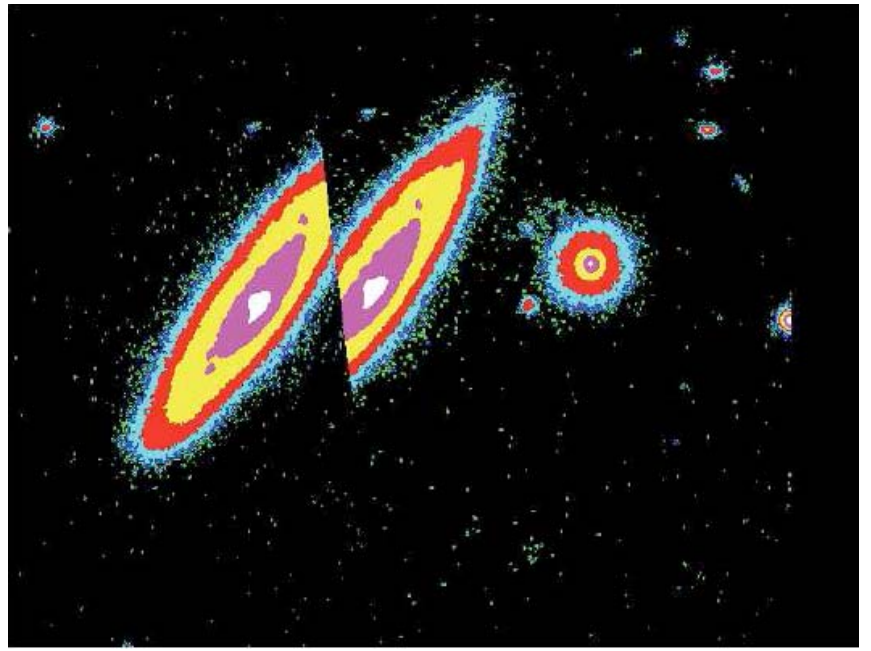

Fig. (7). Third example of a galaxy lensed by acosmic string.

observables is not easy but the hunt appears rewarding: in fact, according to the superstring theory, cosmic strings are the most preferable topological defects to be formed in the early Universe and provide deep insights into the braneworld scenario based on superstrings. More work is required.

\section{ACKNOWLEDGEMENTS}

This work has been partially supported by Russian Foundation for Basic Research grants 10-02-00961a (O.S. and M.S) and grant of the President of RF MK-473.2010.2 and MSU grant for Young Scientists (O.S.). This work has also been partially supported by the Italian Goverment (Ministry of Foreign Affairs).

\section{REFERENCES}

[1] Kibble TWB. Topology of cosmic domains and strings. J Phys A Math Gen 1976; 9: 1387-98.

[2] Zeldovich YaB. Cosmological fluctuations produced near a singularity. Mon Not R Astron Soc 1980; 192: 663.

[3] Vilenkin A. Gravitational field of vacuum domain walls and strings. Phys Rev D Part Fields 1981; 23: 852.

[4] Davis A-C, Kibble TWB. Fundamental cosmic strings. 2005: Avaible from: http://ArXiv.org/hep-th/0505050

[5] Polchinski J. Cosmic super string revised. 2004: Avaible from: http://ArXiv.org/hep-th/0410082v2

[6] Achucarro A, Martins CJAP. Cosmic Strings 2008: Avaible from: http://ArXiv.org/astro-ph/0811.1277v1

[7] Vilenkin A. Shellard EPS. Cosmic strings and other topological defects. Cambridge: Cambridge University Press 1994.

[8] Martins CJAP, Shellard EPS. Fractal properties and small-scale structure of cosmic string networks. Phys Rev D Part Fields 2006; 73: 043515.

[9] Hindmarsh A. In: Gibbons G, Hawking SW, Vachaspathi T, Eds. The formation and evolution of cosmic strings. Cambridge: Cambridge University Press 1990.

[10] Bernardeau F, Uzan J-P. Cosmic string lens phenomenology: Model of Poisson energy distribution. Phys Rev D Part Fields 2001; 63: 023004, 023005.

[11] de Laix AA, Vachaspati T. Gravitational lensing by cosmic string loops. Phys Rev D Part Fields 1996; 54: 4780.

[12] Gangui A, Pogosian L, Winitzki S. Cosmic microwave background bispectrum from active models of structure formation. Phys Rev D 2001; 64: 043001.

[13] Sazhin M, Khovanskaya O, Capaccioli M, et al. Gravitational lensing by cosmic strings: what we learn from the CSL-1 case. Mon Not R Astron Soc 2007; 376: 1731-9.

[14] Kaiser N, Stebbins A. Microwave anisotropy due to cosmic strings. Nature 1984; 310: 391. 
[15] Stebbins A. Cosmic strings and the microwave sky. I - Anisotropy from moving strings. Astrophys J 1988; 327: 584.

[16] Sazhina OS, Sazhin MV, Sementsov VN. Cosmic microwave background anisotropy induced by a moving straight cosmic string. J Exp Theor Phys 2008; 106: 878-87.

[17] Sazhin M, Khovanskaya O, Capaccioli M, et al. Search for Gravitational Lenses Near the Extragalactic Double Object CSL-1. Astron Lett 2005; 31: 73-79. Avaible from: http://ArXiv.org/astro$\mathrm{ph} / 0406516$

[18] Gasparini MA, Marshall P, Treu T, et al. Direct observation of cosmic strings via their strong gravitational lensing effect - I. Predictions for high-resolution imaging surveys. Mon Not R Astron Soc 2008; 385: 1959.

[19] Morganson E, Marshall P, Treu T, et al. Direct observation of Cosmic strings via their strong gravitational lensing effect:II.
Results from HST/ACS image archive. Avaible from: http://ArXiv.org/astro-ph/0908.0602

[20] Vanchurin V, Olum K, Vilenkin A. Cosmic string scaling in flat space. Phys Rev D Part Fields 2005; 72: 063514.

[21] Alcalá JM, Pannella M, Puddu E, et al. The Capodimonte Deep Field. Presentation of the survey and first follow-up studies. Astron Astrophys 2004; 428: 339-352.

[22] Sazhin M, Longo G, Capaccioli M, et al. CSL-1: chance projection effect or serendipitous discovery of a gravitational lens induced by a cosmic string? Mon Not R Astron Soc 2003; 343: 353.

[23] Sazhin, M, Capaccioli M, Longo G, et al. The true nature of CSL1. 2006: Avaible from: http://arxiv.org/abs/astro-ph/0601494

[24] Sazhin M, Capaccioli M, Longo G, Paolillo M, Khovanskaya O. Further spectroscopic observations of the CSL 1 object. Astrophys J 2006; 636: L5-L8.

Received: October 03, 2009

Revised: December 31, 2009

Accepted: January 07, 2010

(c) Sazhin et al.; Licensee Bentham Open.

This is an open access article licensed under the terms of the Creative Commons Attribution Non-Commercial License (http://creativecommons.org/licenses/by-nc/3.0/) which permits unrestricted, non-commercial use, distribution and reproduction in any medium, provided the work is properly cited. 